\title{
Distribution of Dengue Virus Serotypes in Jazan Region, Southwest Saudi Arabia
}

\author{
Ommer Dafalla ${ }^{1 *}$, Abdulaziz Hakami ${ }^{1}$, Elsiddig Noureldin ${ }^{1}$, Siddig Abdelwahab ${ }^{2}$, Yehya \\ Hejri ${ }^{3}$, Tareq Khawaji ${ }^{4}$, Jaber Ghzwani ${ }^{1}$, Omar Mashi ${ }^{1}$, Khalid Shrwani ${ }^{1}$, Tariq Hamzi ${ }^{4}$, \\ Abdullatif Matabi ${ }^{4}$, Abdullah Aghamdi ${ }^{5}$ and Zaki Eisa ${ }^{1}$
}

${ }^{1}$ Saudi Public Health Authority, Jazan,, Saudi Arabia

${ }^{2}$ Substance Abuse Research Centre, Jazan University, Kingdom of Saudi Arabia

${ }^{3}$ Infectious Disease, Public Health, Ministry of Health, Jazan, Saudi Arabia

${ }^{4}$ Department Molecular Biology, King Fahad Central Hospital Ministry of Health, Jazan, Saudi Arabia

${ }^{5}$ Faculty of Science, King Abdulaziz University, Jeddah, Saudi Arabia

\begin{abstract}
Background: Nearly half of the world's population are at risk of dengue fever (DF) which is a serious threat to the majority of international public health. Recently, Dengue viruses (DENVs) considered a re-emerging arthropod-borne viral disease that has been growing its transmission in numerous geographic areas across the world, causing dengue-endemic in more than 125 countries globally.

Methodology/Principal findings: One hundred and ninety-two serum samples were tested to determine the circulating DENV serotype in Jazan region. Serotyping was performed using RT-PCR followed by nested PCR and the results were further confirmed using sequencing. IBM SPSS statistical software, version 22.0, was used in both the descriptive and inferential statistical data analysis. The predominant serotype circulating in Jazan region was DENV-2 (76.6\%), followed by DENV-3 (17.20\%), and DENV-1 (6.3\%). DENV-4 serotype was not detected. The highest occurrence of DENV-1 (19\%) was in the middle sector of Jazan region. While DENV-2 highest occurrence was in Northern sector (85.3\%). On the other hand, DENV-3 highest occurrence was in western sector (27\%). The occurrence percentages of DENV-1 and -3 serotypes did not differ by sector. Likewise no difference in serotypes distribution according to the gender. DENV-2 and -3 were differently distributed amongst various age groups, while DENV-1 showed similar distribution. Bioinformatics analysis for the partial sequencing of RT-PCR products confirmed the results of serotypespecific nested PCR.
\end{abstract}

Conclusions/Significance: The results of this study reported the circulation of the dengue virus types DENV-1, DENV-2, and DENV-3 in Jazan region with the predominance of DENV-2. The study highlights the importance of monitoring and tracking the spread of dengue virus serotypes and their implication for analyzing changes and patterns in dengue endemicity in study areas over time. On-going dengue surveillance to continuously detect outbreaks, along with DENV serotypes and genotypes circulating, and determine dengue fever burden are urgently needed in the region.

\section{Abbreviations}

DF: Dengue Fever; DNVs: Dengue Virus; DHF: Dengue Hemorrhagic Fever; DSS: Dengue Shock Syndrome; WHO: World Health Organization; RNA: Ribonucleic Acid; RT-PCR: Reverse Transcriptase Polymerase Chain Reaction; bp: Base Pair; BLAST: Basic Local Alignment Search Tool; MEGA: Molecular Evolutionary Genetic Analysis; SPSS: Statistical Product and Service Solutions.

\section{Introduction}

Recently, Dengue viruses (DENVs) are considered as reemerging vector transmitted viral disease. More than 125 countries are considered endemic to DENVs [1,2]. Moreover, nearly half of the world's population is at risk of dengue fever (DF) [3]. DENVs cause three spectrum of pathological conditions including dengue fever (DF), Dengue hemorrhagic fever (DHF) and dengue shock syndrome (DSS). DHF and DSS reveal with severe and fatal symptoms [4,5]. These three spectrum are caused by four different serotypes of DENVs (DENVs-1, 2, 3, 4) which are genetically related but

*Corresponding author: Ommer Dafalla, Saudi Public Health Authority, Jazan, Saudi Arabia

Accepted: August 28, 2021

Published online: August 31, 2021

Citation: Dafalla O, Hakami A, Noureldin E, et al. (2021) Distribution of Dengue Virus Serotypes in Jazan Region, Southwest Saudi Arabia. Ann Public Health Reports 5(2):207-215 
antigenically distinct $[6,7]$. There are several factors that may contribute to dengue-epidemics including global climatic aspects such as high temperatures and increasing rainfall rates in addition to unplanned urbanization $[2,8,9]$. DENVs are primarily transmitted by different species of female Aedes mosquitoes; Aedes aegypti, Aedes polynesiensis and Aedes albopictus. Aedes aegypti is known as the foremost viral vector-borne transmitter due to its closeness to human inhabitation $[2,4,10,11]$. In recent decades, the incidence of DENVs has developed intensely around the world [11]. The World Health Organization (WHO, 2020) recorded that around 390 million people test positive for DENVs yearly, of which 96 million of them were clinically ill and presented severe complications $[3,11]$. A study by Bhatt, et al. [12] estimated that 3.9 billion individuals, who resident in the epidemic part of the tropical and subtropical areas are at risk of dengue infection $[2,11,12]$. In spite of a risk of infection prevailing in more than 125 countries, $70 \%$ of the actual disease burden was reported in Asia $[3,13]$. The WHO has reported an 8-fold increase in the number of dengue laboratory-confirmed cases over the last two decades, from 505,430 confirmed cases in 2000, to more than 2.4 and 4.2 million in 2010 and 2019, respectively. A 4-fold increase in Dengue-related deaths was also observed from 960 deaths in the year 2000 to 4032 deaths in 2015 [3]. Several epidemiological studies have reported that Africa, South Africa, Southeast Asia and the Arabian Peninsula are considered the most dengue endemic regions due to high circulation of dengue virus and the presence of its vectors $[10,14]$.
In the Kingdom of Saudi Arabia (KSA), two dengue virus serotypes (DENV-1 and -2) were firstly reported during an outbreak in Jeddah city in 1994 with 289 confirmed cases [13]. DENV-3 serotype was also detected in Jeddah city in $1997[1,2,15,16]$. Following this, several DF outbreaks have been reported in different regions of Saudi Arabia bringing a serious threat to non-infected individuals [2]. Recently in Saudi Arabia, there has been a dramatic rise in the Aedes aegypti mosquitoes population as well as the emerging distribution of many dengue outbreaks [17]. DENVs have been detected in different regions of KSA such as Jeddah, Makkah, Al-Madinah, Jazan and Aseer $[16,18,19]$. In Jazan region, there were 4192 PCR-confirmed dengue cases between 2005 and 2019 with the highest out-breaks occurred in 2019 (1623 cases), followed by 436 cases in 2016 as reported by the Dengue Control Program in Jazan, KSA. There is yet no data that has been published on the distribution of dengue virus serotypes in the 16 provinces of the Jazan region. Hence, this study is considered the first of its kind and designed to investigate dengue virus serotypes distribution in the provinces of Jazan region located in the Southwest of Saudi Arabia.

\section{Methodology}

\section{Study area and sampling}

Jazan region is considered a subtropical climate, and it is located in the southwest of the Kingdom of Saudi Arabia (Figure 1). It lies between $16^{\circ}-12$, and $18^{\circ}-25$, latitude north. It is surrounded by the Red Sea $(260 \mathrm{~km})$ from the west and by Arabic Republic of Yemen $(120 \mathrm{~km})$ from the south and east and Asir region from the north [20].

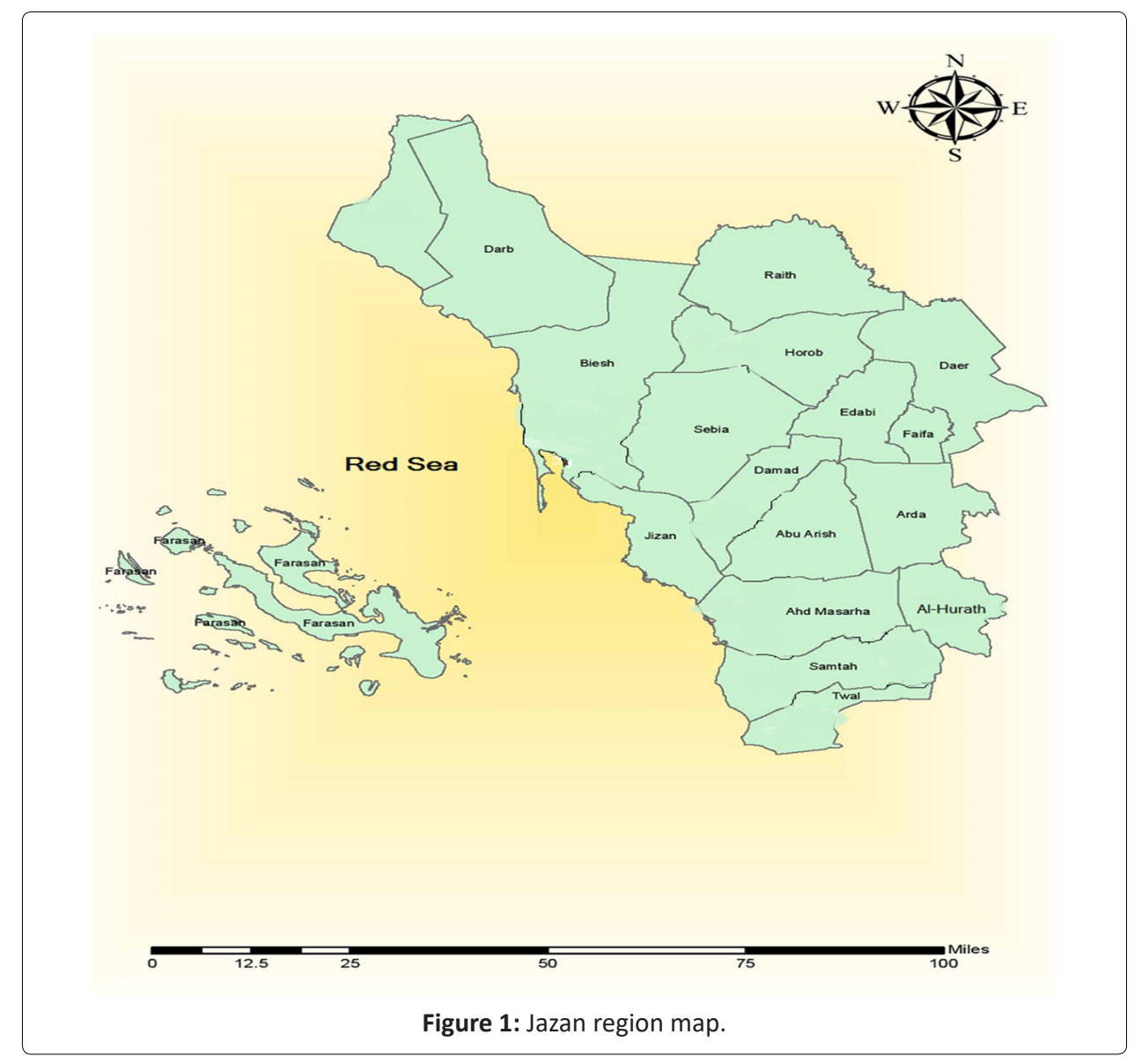


One-hundred ninety two positive dengue serum samples were collected from 16 provinces in Jazan region and distributed at 6 health sectors as shown in Table 1. The use of human subjects for this study was approved by the ethical committee of Saudi Center for Disease Prevention and Control, Jazan, Saudi Arabia (SCDC). Patients were deidentified and study data was analyzed anonymously.

\section{RNA isolation}

GeneJET Viral DNA and RNA Purification Kits were procured from Thermo Scientific (U.S.A) and used to extract RNA follows the manufacturer's instructions.

\section{Reverse transcriptase polymerase chain reaction (RT-PCR)}

The test was performed according to the Lanciotti, et al. 1992 [21]. D1 consensus forward primer (5TCAATATGCTGAAACGCGCGAGAAACCG-3) and D2 revers primer (5-TTGCACCAACAGTCAATGTCTTCAGGTTC-3) synthesized in Integrated DNA Technology (Belgium) were used to amplify 511 base pair of viral genome. One step RT-PCR reactions were performed according to access RT-PCR-system protocol (Thermo Fisher Scientific Inc. Waltham, Massachusetts, United Stateswith) in $50 \mu \mathrm{L}$ volume containing $25 \mu \mathrm{L}$ of $2 \mathrm{XRT}$ PCR buffer, $1 \mu \mathrm{L}$ of 25X RT-PCR Enzyme Mix, $50 \mathrm{pmol}$ (final concentration $1 \mu \mathrm{M}$ ) of each forward (D1) and reverse (D2) primers, $5 \mu$ l of RNA virus and nuclease free water to total volume $50 \mu$ l. The thermal cycling incubations temperatures programmed as follows: incubation for 1 hour at $42^{\circ} \mathrm{C}$ (to transcribed the RNA to cDNA), initial denaturation for 5 minutes at $94^{\circ} \mathrm{C}$ followed by 35 cycle of denaturation $\left(94^{\circ} \mathrm{C}\right.$, for 30 second), primers annealing $\left(55^{\circ} \mathrm{C}\right.$ for 1 minute), primer extension $\left(72^{\circ} \mathrm{C}\right.$ for 2 minutes) and final extension for 5 minutes.

\section{Nested-PCR}

Nested-PCR was done in single tubes for each sample in $50 \mu \mathrm{l}$ reaction mixture containing $25 \mu \mathrm{l} \mathrm{GoTag}{ }^{\circledR} \mathrm{G} 2$ green master mix ready to use from Promega, $5 \mu$ l of the diluted (1:50) RT-PCR product, $50 \mathrm{pmol}$ (final concentration $1 \mu \mathrm{M}$ ) of each consensus forward primer D1 and type specific reverse primers TS1(serotype 1), TS2 (serotype 2), TS3 (serotype 3) and TS4 (serotype 4) "Table 2". The thermal cycles conditions, initial denaturation for 3 minutes at $94^{\circ} \mathrm{C}$ followed by 30 cycles of denaturation $\left(94^{\circ} \mathrm{C}, 30 \mathrm{~s}\right)$, annealing temperature $55^{\circ} \mathrm{C}$ for $1 \mathrm{~min}$, primer extension $\left(72^{\circ} \mathrm{C}, 2 \mathrm{~min}\right)$ and final extension for 5 minutes. In each run negative and positive controls were included. The PCR products of nested amplification were analyzed by gel electrophoresis ( $2 \%$ agarose in TrisAcetate EDTA buffer) staining with ethidium bromide. The

Table 1: Distribution of governates based on health sectors.

\begin{tabular}{|l|l|}
\hline Health sector & Governates \\
\hline Central sector & Jizan \\
\hline Northern sector & Raith, Biesh and Darb \\
\hline Western sector & Sabyah, Damad, Horob \\
\hline Middle sector & Abuarish, Ardah \\
\hline Southern sector & AhdMasarha, Twal, Samtah, Al-Hurath \\
\hline Mountainous sector & Edabi, Faifa, Daer \\
\hline
\end{tabular}

Table 2: Primers used for dengue serotyping.

\begin{tabular}{|l|l|l|l|}
\hline Primer & Sequence 5 - 3 & $\begin{array}{l}\text { Genome } \\
\text { position }\end{array}$ & Size in bp \\
\hline TS1 & CGTCTCAGTGATCCGGGGG & $568-586$ & 482 \\
\hline TS2 & CGCCACAAGGGCCATGAACAG & $232-252$ & 119 \\
\hline TS3 & TAACATCATCATGAGACAGAGC & $400-421$ & 290 \\
\hline TS4 & CTCTGTTGTCTTAAACAAGAGA & $506-527$ & 392 \\
\hline
\end{tabular}

visualization was carried out using Gel Doc XR Imaging System (Bio-Rad).

\section{Sequencing and bioinformatics analysis}

Single-pass sequencing was performed on RT-PCR products using D1 primer (ten samples from each serotype) by Macrogen Company (Seoul, Korea) to confirm nested-PCR results. The sequences were searched for sequence similarity through Basic Local Alignment Search Tool (BLAST) (www. ncbi.nlm.nih.gov/BLAST/) [22] and compared to reference sequences of Dengue serotypes published in BLAST (www. ncbi.nlm.nih.gov/genbank/). Evolutionary relationship of taxa tree constructed for each serotype using the Molecular Evolutionary Genetic Analysis (MEGA 5).

\section{Data analysis}

Data was entered in Microsoft Excel software. IBM SPSS statistical software, version 22.0, and analyzed for the descriptive and inferential statistical outcomes. The study variables were categorically presented as a percentage for descriptive analysis. For inferential statistics, chi square test of homogeneity was used to compare the various percentage distributions of DENV serotypes among gender, areas and age groups. The cutoff point for the significant level (alpha error) was set as 0.05 .

\section{Results}

Results of RT-PCR and nested PCR showed that three serotypes are circulating in Jazan region; DENV-2 (76.6\%), followed by DENV-3 (17.20\%), and DENV-1 (6.3\%). DENV-4 serotype was not detected (Figure 2).

\section{Blast analysis results}

The BLAST analysis for the partial sequencing of RTPCR products confirmed the results of serotype-specific nested PCR, and sequences results aligned along with many sequences of dengue virus serotypes were published in the Gen bank. The evolutionary relationship of taxa constructed by the MEGA5 program (Figure 3, Figure 4, Figure 5) shows the relationship between the varieties of the dengue virus serotype 1,2,3 detected in Jazan region and some previously published datasets selected from GenBank.

\section{Statistical analysis results}

Chi square statistical test showed that the proportions of DENV serotypes are statistically significant (Chi square = 164.906, $P<0.05)$. The distribution of these serotypes also investigated within geographical areas, gender and age groups. As shown in Table 3, the highest occurrence of DENV$1(19 \%)$ was in the middle sector of Jazan region. While DENV- 
Citation: Dafalla O, Hakami A, Noureldin E, et al. (2021) Distribution of Dengue Virus Serotypes in Jazan Region, Southwest Saudi Arabia. Ann Public Health Reports 5(2):207-215

Table 3: Serotypes distribution by sector.

\begin{tabular}{|l|c|c|c|c|}
\hline Sectors & Serotype 1 & Serotype 2 & Serotype 3 & Total \\
\hline Central sector & $3(7,1 \%)$ & $32(76,2 \%)$ & $7(16,7 \%)$ & $42(22 \%)$ \\
\hline Northern sector & $1(2,9 \%)$ & $29(85,3 \%)$ & $4(11,8 \%)$ & $34(17,7 \%)$ \\
\hline Western sector & $1(2,1 \%)$ & $33(70,2 \%)$ & $13(27,7 \%)$ & $47(24,5 \%)$ \\
\hline Middle sector & $4(19 \%)$ & $13(62 \%)$ & $4(19 \%)$ & $21(10,8 \%)$ \\
\hline Southern sector & $3(6.3 \%)$ & $40(83,3 \%)$ & $5(10,4 \%)$ & $48(25 \%)$ \\
\hline Mountainous sector & 0 & 0 & 0 & 0 \\
\hline Total & $12(6,2 \%)$ & $147(76,6 \%)$ & $33(17,2 \%)$ & 192 \\
\hline Chi square (P-value) & $3.0(0.558)$ & $13.646(0.009)$ & $8.667(0.07)$ & \\
\hline
\end{tabular}

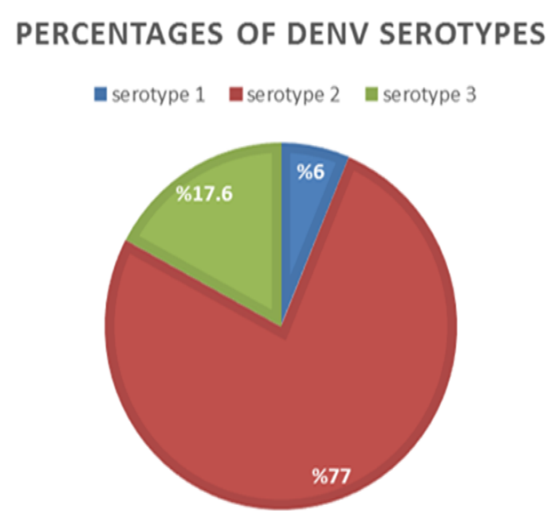

Figure 2: The distribution of DENV serotypes detected in serum samples collected from Jazan region during 2020.

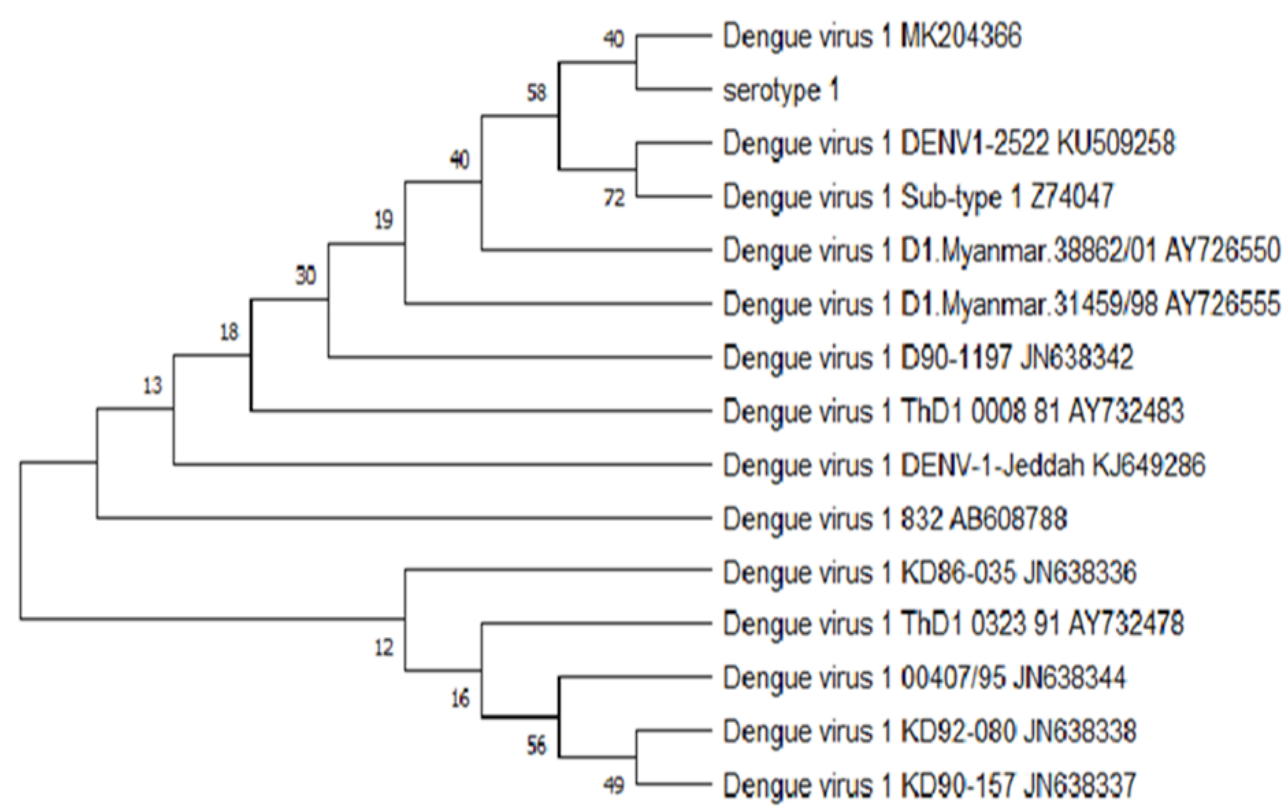

Figure 3: Evolutionary relationship of taxa for dengue virus serotype 1 samples detected in Jazan region (sample 1).

2 highest occurrence was in Northern sector (85.3\%). On the other hand, DENV-3 highest occurrence in western sector (27\%). The percentages of DENV 1 and 3 serotypes did not differ by sector $(P>0.05)$. Chi square test of homogeneity showed that there is a significant difference in the distribution of DENV2 serotypes between the various sectors $(P<0.05)$.
The three serotypes were equally distributed between female and male population $(P>0.05)$ as shown in Table 4.

Table 5 depicted that DENV 2 and 3 are differently distributed amongst various age groups, while DENV 1 showed similar distribution $(P>0.05)$. And Figure 6 illustrated the distribution of dengue virus samples by age groups. 


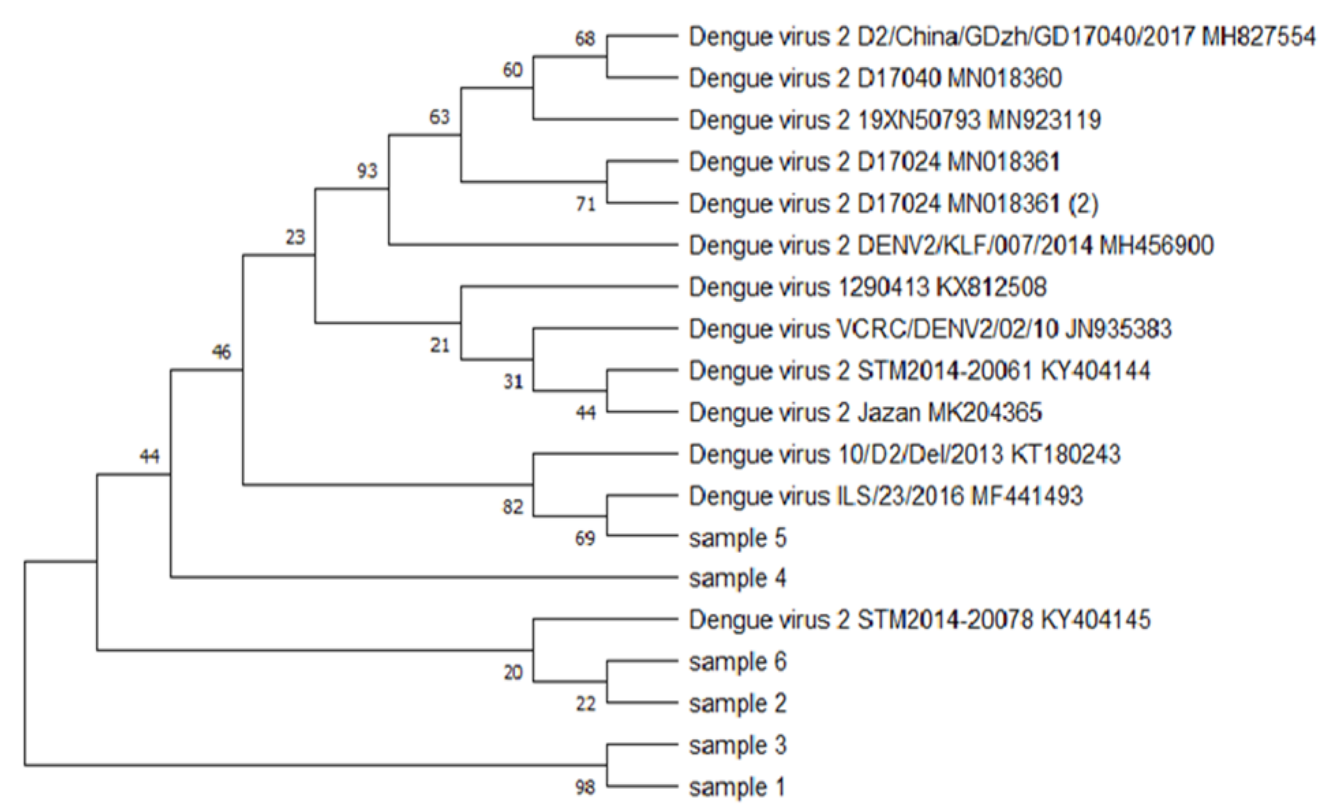

Figure 4: Evolutionary relationship of taxa for dengue virus serotype 2 samples detected in Jazan region (samples 1, 2, 3, 4, 5, 6).

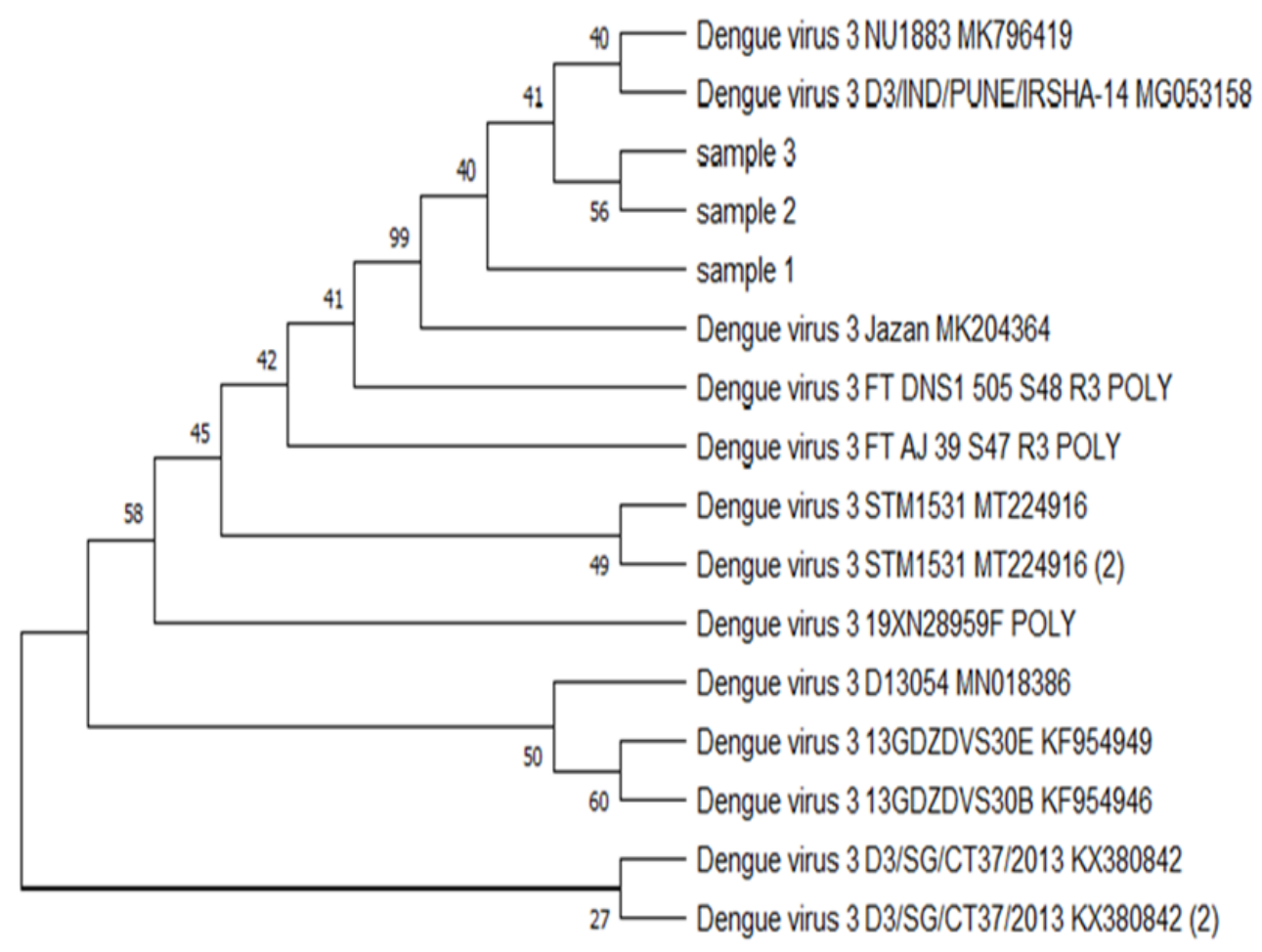

Figure 5: Evolutionary relationship of taxa for dengue virus serotype 3 samples detected in Jazan region (samples 1, 2, 3).

Table 4: Serotypes distribution by gender.

\begin{tabular}{|l|c|c|c|c|}
\hline & Serotype 1 & Serotype 2 & Serotype 3 & Total \\
\hline Male & $7(6,4 \%)$ & $84(77,1 \%)$ & $18(16,5 \%)$ & $109(56,8 \%)$ \\
\hline Female & $5(6 \%)$ & $63(76 \%)$ & $15(18 \%)$ & $83(43.2 \%)$ \\
\hline Total & $12(6,2 \%)$ & $147(76,6 \%)$ & $33(17,2 \%)$ & 192 \\
\hline
\end{tabular}


Citation: Dafalla O, Hakami A, Noureldin E, et al. (2021) Distribution of Dengue Virus Serotypes in Jazan Region, Southwest Saudi Arabia. Ann Public Health Reports 5(2):207-215

Table 5: Serotypes distribution by age groups.

\begin{tabular}{|l|c|c|c|c|}
\hline Age group & Serotype 1 & Serotype 2 & Serotype 3 & Total \\
\hline $0-10$ years & $1(8.3 \%)$ & $8(66.7 \%)$ & $3(25 \%)$ & $12(6.3 \%)$ \\
\hline $11-20$ years & $2(6.25 \%)$ & $26(81.25 \%)$ & $4(12.5 \%)$ & $32(16.7 \%)$ \\
\hline $21-40$ years & $7(7.7 \%)$ & $67(73.6 \%)$ & $17(18.7 \%)$ & $91(47.4)$ \\
\hline $41-60$ years & $2(4.2 \%)$ & $41(85.4 \%)$ & $5(10.4 \%)$ & $48(25 \%)$ \\
\hline over 60 years & $0(0 \%)$ & $5(55.6 \%)$ & $4(44.4 \%)$ & $9(4.7 \%)$ \\
\hline Total & $12(6.2 \%)$ & $147(76.6 \%)$ & $33(17.2 \%)$ & 192 \\
\hline Chi square $(P$-value) & $7.33(0.062)$ & $88.88(0.00)$ & $20.78(0.00)$ & \\
\hline
\end{tabular}

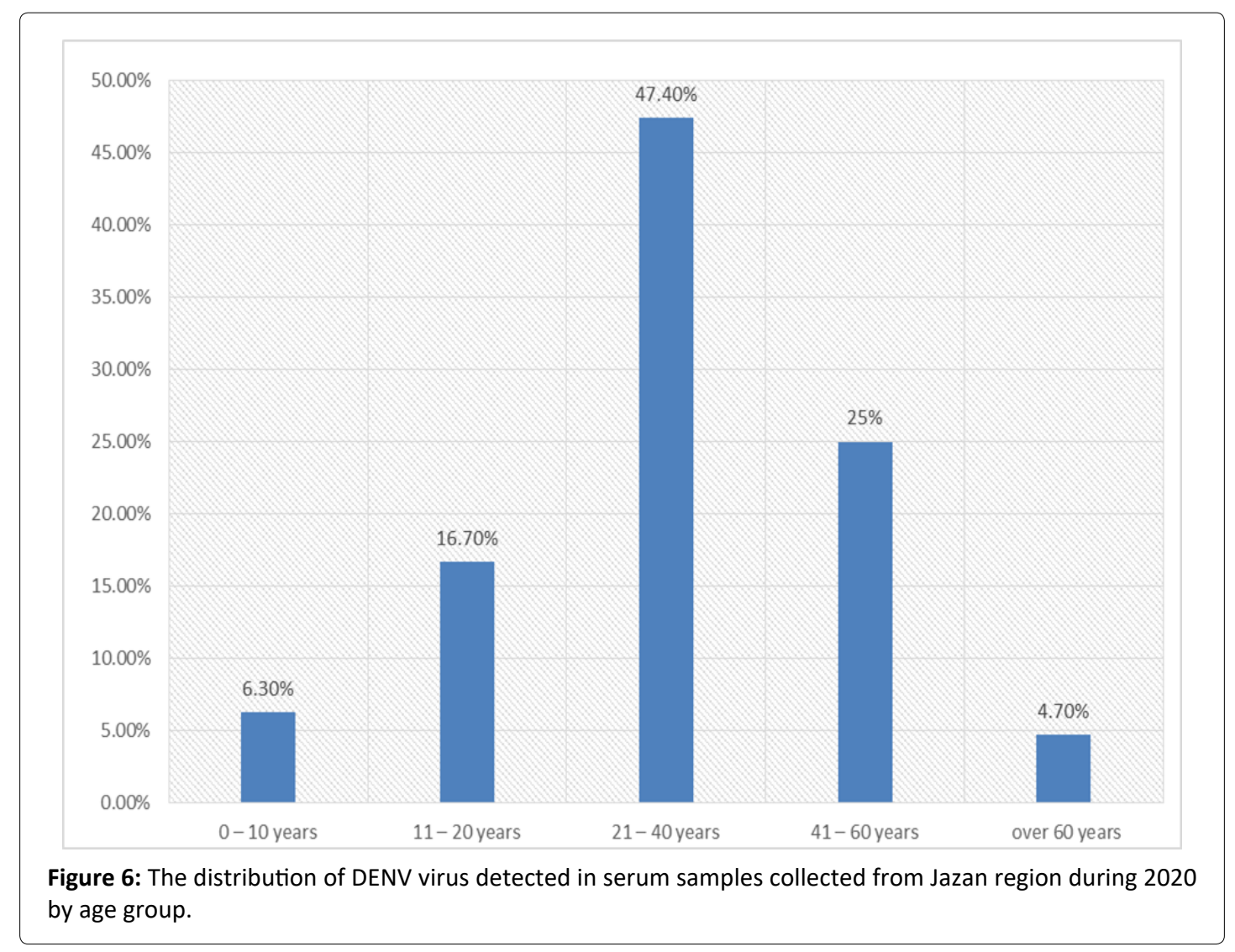

\section{Discussion}

The current study was designed to investigate the distribution of DENV viruses collected from Jazan region using RT-PCR, sequencing and bioinformatic analysis. Many sporadic dengue outbreaks have been recorded in Jazan region during the recent decade (290 cases in 2010, 289 in 2012, 436 in 2016, 320 in 2017, 174 in 2018, and 1653 in 2019 as reported by Dengue Control Program in Jazan. Some serological surveys have been conducted in the region and revealed dengue prevalence of $26.5 \%$ and $47.74 \%$, respectively $[18,23]$. However, dengue fever had become highly prevalent in Jazan region $(56.4 \%)$ compared to the previous reports [2]. The results of this study showed that three dengue virus types (DENV-1, DENV-2 and DENV-3) were circulating in Jazan region with the predominance of DENV-2 (76.6\%), followed by DENV-3 (17.20\%), and DENV-1 (6.3\%). DENV-4 serotype was not detected (Figure 2). These findings are slightly different from our previous study where the DENV-2 rated 83.9\%, DENV-1 (8.9\%), then DENV-3 (7.2\%) [2].
Changes and shifts in serotypes (DENV-1, DENV-2 and DENV3) between 2016 and 2020 in Jazan region is thought to be a result of failure in heterotypic immunity by antibody induced by the first predominantly circulating serotype (DEN-2) in the population. Consequently, in Jazan region, it is suggested that DENV-3 and DENV-1 might have been recently introduced and spread in a population that had immunity mainly to the predominant DENV-2 [24]. The finding is in partial accordance with the results of Fakeeh and Zaki (2001) and Ashishi (2017) in Jeddah, Saudi Arabia, who found that DENV-2 was the predominant serotype, followed by DENV-1, and DENV-3 $[15,25]$. While Organji ,et al. (2017) in Makkah city, Saudi Arabia, recorded that DENV-1 was the predominant dengue virus type, followed by DENV-2 and then DENV-3 in contrast to the prevalence of DENV-2 found by Khan, et al. [1,26]. In Sabya Governate of Jazan region, the circulating of both DENV-2 and DENV-3, With DENV-2 being the most common and predominant type in tested area (DENV-2 69.7\% and DENV-3 30.3\%) [27]. 
DENV-1 and DENV-2 caused the major outbreak in 1994 in Jeddah, while DENV-3 emerged in 1997 [28]. In addition, two genotypes for DENV-1 (America Africa genotype, and Asia-2 genotype), DENV-2 genotype clustered within Cosmopolitan genotype, and DENV-3 clustered within genotype III, were reported from Jeddah by Zaki, et al. at 2008 [28]. Hashem, et al. [29] showed that genotype III isolates of DENV-3 are the only circulated genotype in Jeddah and it was in close similarity to Singapore isolate suggesting the possible role of pilgrims in DENV-3 importation into western Saudi Arabia during Hajj and Umrrah seasons [29]. The DENV-2 isolate from Jeddah, which belongs to the Cosmopolitan genotype, was found genetically related to isolates in Pakistan circulating from 2008 to 2013 [30]. It seems that the importation of DENV-2 isolates into Saudi Arabia between 1992 and 2014 was mainly due to the highest numbers of pilgrims attending either Hajj or Umrrah pilgrimages from endemic countries like Indonesia, Pakistan, and India. The three dengue virus serotypes DENV-1, DENV2 , and DENV-3 are circulating in the Middle East countries, including Saudi Arabia and Yemen [5]. Moreover, DENV-4 was reported for the first time from asymptomatic blood donners in Jeddah, Western region of Saudi Arabia in 2017 [25]. The ranking of the prevalence of dengue virus serotypes in Jeddah was DENV-2 (48\%), DENV-4 (30\%), DENV-1 (20\%) and DENV-3 (2\%). In Singapore, the prevalence of DENV-1 to DENV-4 for residents aged $16-60$ years was found to be $41.9 \%, 47.8 \%$, $24.7 \%$, and $16.0 \%$, respectively [31].

However, a study found that cases infected by DENV2 had a higher proportion of severe dengue than those of DENV-1 and DENV-4 [32]. Furthermore, it is well known that, in secondary infection, especially infections i.e. DENV-2 followed by DENV-3/DENV-1 or DENV-3 followed by DENV-2 may result in severe dengue complications. Thus, any future outbreak with DENV-1/DENV-3 serotype may cause high morbidity or mortality in Jazan region. Therefore, enhanced dengue surveillance operations should be deployed, especially in the rain and post-rain periods to prevent severe, and large-scale dengue outbreaks in the region [33]. Early detection of serotypes circulating in Jazan region could be an important approach to prevent increasing numbers of severe complications during dengue outbreaks. This could help the health predicting support needed for early diagnoses and treatment of dengue cases [32]. Consequently, the primary infections by DENV-1 and DENV-3 are known to be related with more dengue severe infections, while infections with DENV-2 and DENV-4 are associated with increased dengue severity only if they present as secondary infections [34]. These data show the importance of continuous monitoring and evaluating of the circulated DENVs in Saudi Arabia to take effective control and management strategies in the Kingdom at earlier. Constant and sustainable screening of the pilgrims of Hajj and Umrrah at the ports is imperative to prevent the introduction of new DENV strains [30].

The increment of dengue cases and the circulating of the three dengue virus types in Jazan region may be attributed to several factors; traveling abroad of Jazan citizens to dengue endemic countries or locally for Hajj and Umrrah or for trade or other purposes, proximity of Jazan region to Yemen where the disease is endemic and the three dengue virus serotypes DENV-1, DENV-2, and DENV-3 are circulating, climatic conditions, increasing urbanization, socioeconomic level of the population, and migrant labors from all over the world especially those from dengue endemic countries.

As shown in Table 3, the diversity of serotype prevalence within different areas may be due to the differences in the genetics and immunity systems of population at every sector. Additionally, the collective occurrence of the three serotypes in each of the southern sector $(25 \%)$, western sector $(24.5 \%)$ and central sector (22\%) may be described as due to the nature of those sectors which are relatively flat and permit for the formation of small stagnant water collections following the rainfall [18]. Nonetheless, the circulation of the three serotypes in the different geographical sectors may determine the overall severity of the disease.

DENV-3 and DENV-1 were probably imported into Jazan region from other parts of the Kingdom or foreign countries. Unlike the predominant DENV-2, population of Jazan region may have no sufficient herd immunity against DENV-3 and DENV-1. Interestingly in this study, no dengue cases were reported from the mountainous sector. This may be attributed to the fact that their altitude range is more than 1800-2000 meters above sea level, whereas Aedes aegypti range of vertical flight is commonly encountered up to 1,700 $\mathrm{m}$, but rare above that level [35].

In the present study, although the percentage of male samples is more than females (56.8\% and $43.2 \%$, respectively), the three serotypes were equally distributed between female and male population $(P>0.05)$ as shown in Table 4. This result is in accordance with the work of Kalayanarooj and Nimmannitya (2000) who reported no gender difference [36]. However, it contradicted with some studies, which demonstrated that male gender and young age are potential risk factors for dengue fever in Saudi Arabia $[18,26,37,38]$. The finding is also inconsistent with three other studies conducted in Australia, France and Mexico, which revealed that dengue fever was more distributed among the female population [39-41]. Table 5 depicted that DENV 2 and 3 are differently distributed amongst various age groups, while DENV 1 showed similar distribution ( $P>0.05$ ). In Indonesian pediatric population, it was noted that more than half $(50.9 \%)$ had already been exposed to more than one dengue serotype, and that proportion increased with age [42]. In the present study the 21-40 years was the highest age group exposed to dengue infection by all three serotypes $(47.4 \%)$, followed by $41-60$ years age group $(25 \%)$ and then $11-20$ years age group (16.7\%). While youngest and eldest age groups ( $0-10$ years and over 60 years) produced the least dengue incidence, $6.3 \%$ and $4.7 \%$, respectively. This result is fair if we consider that the majority of Saudi population (66.4\%) is adult and youth (15-54 age group), followed by youngest $24.7 \%$ (<15 years), elder $5.7 \%$ (55-64 age group), and eldest $3.2 \%$ (> 65 years) [43]. The adult and young (2160 years) were always involved in outdoor activities which in turn increased their chances to encounter the virus by the bites of infective Aedes aegypti. 


\section{Conclusion}

In conclusion, the results of this study indicated the circulation of the dengue virus types DENV-1, DENV-2, and DENV-3 in Jazan region with the predominance of DENV-2. The study highlights the importance of monitoring and tracking the spread of dengue virus serotypes and their implication for analyzing changes and patterns in dengue endemicity in study areas over time. On-going dengue surveillance to continuously detect outbreaks, along with DENV serotypes and genotypes and determining the dengue fever burden are urgently needed in the region. Multiple circulated serotypes should rigorously be monitored as it may lead to increased cases of severe form of dengue

\section{Data Availability}

The data used to support the findings of this study are available from the National Center for Disease Prevention and Control, Saudi Arabia (SCDC).

\section{Declarations}

\section{Ethical approval}

The use of human subjects for this study was approved by the ethical committee of Saudi Center for Disease Prevention and Control, Jazan, Saudi Arabia (SCDC). Patients were deidentified and study data was analyzed anonymously.

\section{Consent for publication}

Not applicable.

\section{Competing interests}

The authors declare that they have no competing interests regarding the publication of this manuscript.

\section{Funding}

Not applicable.

\section{Authors' contributions}

$\mathrm{OD}, \mathrm{TK}, \mathrm{JM}, \mathrm{OM}, \mathrm{TH}, \mathrm{AM}$ and $\mathrm{AH}$ participated in samples collection and carried out the experimental work. OD, EN, SA and ZE participated in the design of the study, reviewing, analysis of the results, Data curation, validation, reviewing \& editing. $O D, E N, S A$ and $K S$ wrote the manuscript. $Y H, A G$, $\mathrm{KS}, \mathrm{AH}$ and $\mathrm{MB}$ participated in Data curation reviewing and validation. All authors read and approved the final manuscript.

\section{Acknowledgements}

This work was supported by the Saudi Center for Disease Prevention and Control (SCDC). We thank the clinical and other support staff at the study sites for their cooperation and assistance.

\section{Author summary}

Recently, Dengue viruses (DENVs) considered a reemerging arthropod-borne viral disease has been growing its transmission in numerous geographic areas across the world, causing dengue-endemic in more than 125 countries globally. Serum samples for this study were collected from
Jazan region, southwest Saudi Arabia. One-hundred ninety two dengue positive serum samples were serotyped using RT-PCR followed by Nested PCR and the results confirmed by sequencing and bioinformatics analysis. The predominant serotype in Jazan region was DENV-2 (76.6\%), followed by DENV-3 (17.20\%), and DENV-1 (6.3\%). DENV-4 serotype was not detected. The distribution of these serotypes was also investigated within geographical areas, gender and age groups. The highest occurrence of DENV-1 (19\%) was in the middle sector of Jazan region, DENV-2 highest occurrence was in Northern sector $(85,3 \%)$ and DENV-3 highest occurrence was in western sector (27\%). The three serotypes were equally distributed between female and male population. DENV 2 and 3 were differently distributed amongst various age groups, while DENV 1 showed similar distribution. This study confirmed the circulation of three dengue virus serotypes (DENV-1, DENV-2 and DENV-3) and highlighted the importance of their monitoring and surveillance for tracking the dengue virus circulating serotypes. The current findings could be used by the officials in the surveillance, prevention, and control programs.

\section{References}

1. Organji SR, Abulreesh HH, Osman GEH (2017) Circulation of dengue virus serotypes in the city of makkah, saudi arabia, as determined by reverse transcription polymerase chain reaction. Can J Infect Dis Med Microbiol 2017: 1646701.

2. Alsheikh AA, Daffalla OM, Noureldin EM, et al. (2017) Serotypes of dengue viruses circulating in Jazan region, Saudi Arabia. Biosci Biotech Res Comm 10: 11-21.

3. World Health Organization (2021) Dengue and severe dengue.

4. El-Gilany AH, Eldeib A, Hammad S (2010) Clinico-epidemiological features of dengue fever in Saudi Arabia. Asian Pac J Trop Med 3: 220-223.

5. Nedjadi T, El-Kafrawy S, Sohrab SS, et al. (2015) Tackling dengue fever: Current status and challenges. Virol J 12: 212.

6. (1997) Dengue haemorrhagic fever, diagnosis, treatment, prevention and control. ( $2^{\text {nd }}$ edn) WHO Geneva, 48-59.

7. Holmes EC, Twiddy SS (2003) The origin, emergence and evolutionary genetics of dengue virus. Infect Genet Evol 3: 19-28.

8. Chowell G, Cazelles B, Broutin H, et al. (2011) The influence of geographic and climate factors on the timing of dengue epidemics in Perú, 1994-2008. BMC Infect Dis 11: 164.

9. Banu S, Hu W, Hurst C, et al. (2011) Dengue transmission in the Asia-Pacific region: Impact of climate change and socioenvironmental factors. Trop Med Int Health 16: 598-607.

10. Gubler DJ (2002) Epidemic dengue/dengue hemorrhagic fever as a public health, social and economic problem in the 21st century. Trends Microbiol 10: 100-103.

11. Khetarpal N, Khanna I (2016) Dengue fever: Causes, complications, and vaccine strategies. J Immunol Res 2016: 6803098.

12. Bhatt S, Gething PW, Brady OJ, et al. (2013) The global distribution and burden of dengue. Nature 496: 504-507.

13. Brady OJ, Gething PW, Bhatt S, et al. (2012) Refining the global spatial limits of dengue virus transmission by evidence-based consensus. PLoS Negl Trop Dis 6: e1760. 
14. Heilman JM, De Wolff J, Beards GM, et al. (2014) Dengue fever: A Wikipedia clinical review. Open Med 8: e105-e115.

15. Fakeeh M, Zaki AM (2001) Virologic and serologic surveillance for dengue fever in Jeddah, Saudi Arabia, 1994-1999. Am J Trop Med Hyg 65: 764-767.

16. Alhaeli A, Bahkali S, Ali A, et al. (2016) The epidemiology of Dengue fever in Saudi Arabia: A systematic review. J Infect Public Health 9: 117-124.

17. Aziz AT, Al-Shami SA, Mahyoub JA, et al. (2014) An update on the incidence of dengue gaining strength in Saudi Arabia and current control approaches for its vector mosquito. Parasites \& Vectors 7: 258

18. Al-Azraqi TA, El Mekki AA, Mahfouz AA (2013) Seroprevalence of dengue virus infection in Aseer and Jizan regions, Southwestern Saudi Arabia. Trans R Soc Trop Med Hyg 107: 368-371.

19. Al-Saeed MS, El-Kafrawy SA, Farraj SA, et al. (2017) Phylogenetic characterization of circulating dengue and alkhumra hemorrhagic fever viruses in western Saudi Arabia and lack of evidence of Zika virus in the region: A retrospective study, 20102015. J Med Virol 89: 1339-1346.

20. Al-Sheik AA (2011) Larval habitat, ecology, seasonal abundance and vectorial role in malaria transmission of Anopheles arabiensis in Jazan Region of Saudi Arabia. J Egypt Soc Parasitol 41: 615-634.

21. Lanciotti RS, Calisher CH, Gubler DJ, et al. (1992) Rapid detection and typing of dengue viruses from clinical samples by using reverse transcriptase-polymerase chain reaction. J Clin Microbiol 30: 545-551.

22. Altschul SF, Madden TL, Schäffer AA, et al. (1997) Gapped BLAST and PSI-BLAST: A new generation of protein database search programs. Nucleic Acids Res 25: 3389-3402.

23. Gmail MA, Eisa ZM, Eifan SA, et al. (2014) Prevalence of dengue fever in Jizan area, Saudi Arabia. J Pure Appl Microbiol 8: 225231.

24. Kotaki T, Yamanaka A, Mulyatno KC, et al. (2014) Continuous dengue type 1 virus genotype shifts followed by co-circulation, clade shifts and subsequent disappearance in Surabaya, Indonesia, 2008-2013. Infection, Genetics and Evolution 28: 48-54.

25. Ashshi AM (2017) The prevalence of dengue virus serotypes in asymptomatic blood donors reveals the emergence of serotype 4 in Saudi Arabia. Virol J 14: 107.

26. Khan NA, Azhar El, El-Fiky S, et al. (2008) Clinical profile and outcome of hospitalized patients during first outbreak of dengue in Makkah, Saudi Arabia. Acta Trop 105: 39-44.

27. Mohammed M, Abakar AD, Bakri YM Nour, et al. (2018) Molecular surveillance of dengue infections in Sabya governate of Jazan region, Southwestern Saudi Arabia. Int J Mosq Res 5: 125-132.
28. Zaki A, Perera D, Jahan SS, et al. (2008) Phylogeny of dengue viruses circulating in Jeddah, Saudi Arabia: 1994 to 2006. Trop Med Int Health 13: 584-592.

29. Hashem AM, Sohrab SS, El-Kafrawy SA, et al. (2018) Diversity of dengue virus-3 genotype III in Jeddah, Saudi Arabia. Acta Trop 183: $114-118$.

30. El-Kafrawy SA, Sohrab SS, Ela SA, et al. (2016) Multiple introductions of dengue 2 virus strains into Saudi Arabia from 1992 to 2014. Vector Borne Zoonotic Dis 16: 391-399.

31. Low S, Lam S, Wong W, et al. (2015) Dengue seroprevalence of healthy adults in Singapore: Serosurvey among blood donors, 2009. Am J Trop Med Hyg 93: 40-45.

32. Vicente CR, Herbinger K, Fröschl G, et al. (2016) Serotype influences on dengue severity: A cross-sectional study on 485 confirmed dengue cases in Vitória, Brazil. BMC Infect Dis 16: 320.

33. Das M, Borkakoty B, Sarma K, et al. (2018) Molecular characterisation and phylogenetic analysis of dengue outbreak in Pasighat, Arunachal Pradesh, Northeast India. Indian J Med Microbiol 36: 37-42.

34. Balmaseda A, Hammond SN, Pérez L, et al. (2006) Serotypespecific differences in clinical manifestations of dengue. Am J Trop Med Hyg 74: 449-456.

35. Lozano-Fuentes $\mathrm{S}$, Hayden $\mathrm{MH}$, Welsh-Rodriguez $\mathrm{C}$, et al. (2012) The dengue virus mosquito vector Aedes aegypti at high elevation in Mexico. Am J Trop Med Hyg 87: 902-909.

36. Kalayanarooj S, Nimmannitya S (2000) Clinical and laboratory presentations of dengue patients with different serotypes. WHO 24: 53-59.

37. Alzahrani AG, Al Mazroa MA, Alrabeah AM, et al. (2013) Geographical distribution and spatio-temporal patterns of dengue cases in Jeddah Governorate from 2006-2008. Trans R Soc Trop Med Hyg 107: 23-29.

38. Ayyub M, Khazindar AM, Lubbad EH, et al. (2006) Characteristics of dengue fever in a large public hospital, Jeddah, Saudi Arabia. J Ayub Med Coll Abbottabad 18: 9-13.

39. Horta MA, Bruniera R, Ker F, Catita C, et al. (2014) Temporal relationship between environmental factors and the occurrence of dengue fever. Int J Environ Health Res 24: 471-481.

40. García-Gutiérrez MD, Romero-Zepeda H, Salvador RomeroMárquez R (2013) Risk factors associated to the epidemic outbreak of dengue virus infection. Rev Med Inst Mex Seguro Soc 51: 628-634.

41. Raza FA, Rehman Su, Khalid R, et al. (2014) Demographic and clinico-epidemiological features of dengue fever in Faisalabad, Pakistan. PLoS One 9: e89868.

42. Sasmono RT, Taurel AF, Prayitno A, et al. (2018) Dengue virus serotype distribution based on serological evidence in pediatric urban population in Indonesia. PLoS Negl Trop Dis 12: e0006616.

43. (2020) Population of Saudi Arabia. Fanack Home, Saudi Arabia.

DOI: $10.36959 / 856 / 520$

Copyright: (c) 2021 Dafalla O, et al. This is an open-access article distributed under the terms of the Creative Commons Attribution License, which permits unrestricted use, distribution, and reproduction in any medium, provided the original author and source are credited. 